\title{
Coverings and axions: Topological characterizing of the energy coverings in space-time
}

\author{
Mario Ramírez ${ }^{1}$, Luis Ramírez ${ }^{2}$, Oscar Ramírez ${ }^{3}$, Francisco Bulnes ${ }^{4, ~ * ~}$ \\ ${ }^{1}$ Dept. of Mathematics, ESIME-Azcapotzalco, Mexico City, Mexico \\ ${ }^{2}$ Dept. of Mathematics, UNAM-FES-Aragón, Mexico City, Mexico \\ ${ }^{3}$ Dept. of Mathematics, UNAM-FES-Acatlán, Mexico City, Mexico \\ ${ }^{4}$ Research Dept. in Mathematics and Eng., TESCHA, Chalco, Mexico

\section{Email address:} \\ mramirezf@ipn.mx (M. Ramírez), elluis_47@yahoo.com.mx (L. Ramírez), intermezzoadagio@hotmail.com.mx (O. Ramírez), \\ francisco.bulnes@tesch.edu.mx (F. Bulnes)
}

\section{To cite this article:}

Mario Ramírez, Luis Ramírez, Oscar Ramírez, Francisco Bulnes. Coverings and Axions: Topological Characterizing of the Energy Coverings in Space-Time. Pure and Applied Mathematics Journal. Special Issue: Integral Geometry Methods on Derived Categories in the Geometrical Langlands Program. Vol. 3, No. 6-2, 2014, pp. 6-11. doi: 10.11648/j.pamj.s.2014030602.12

\begin{abstract}
Inside the QFT and TFT frame is developed a geometrical and topological model of one wrapping energy particle or "axion" to establish the diffeomorphic relation between space and time through of universal coverings. Then is established a scheme that relates both aspects, time and space through of the different objects that these include and their spectrum that is characterized by their wrapping energy.
\end{abstract}

Keywords: Axion, Diffeomorphism, Spectrum, Universal Covering, Wrapping Energy

\section{Introduction}

The study begins with a interesting question, how to arrive to a definition of time or energy? We know that the concept with that works the physics and after the mathematics not start of the nothing, are abstractions realized to start of the observation and after interpretation of all that us is sensible. The man has had as concerns the description of that happens in the nature and has mean importance the time and the energy.

To arrive to a definition not has been possible, the intent have been probable, nevertheless, the advances in topological theory of strings and $D$ - branes, furthermore of the obtaining of concepts as curvature and torsion energy, inclusive used in some dissertations as [1] to their application of devices to curvature measure of quantum gravity have done probable the crating of a theory at least to conjecture level of the equivalence of these concepts. Possibly and with the development of photonic and spintronic devices (energy and time established in the QFT through bosons and spins) can be demonstrated under observational facts such theory.

To the question, is possible to establish equivalence between energy and time? Could have as immediate response and categorist no! Nevertheless re-evaluating the question in the context of the ramified field, could establish an equivalence, not in a purely algebraic sense and the continuous applications only, but, if for certain differential applications that born of several dualities (for example, the Langlands duality $[2,3]$ ) and that are deformable images of certain applications between of differential operators in a holomorphic context and whose actions on said elements (ramifications) are actions from of loop groups obtained in the construction of cycles of the space-time [3].

\section{Spectrum of the Commutative Rings}

Let $X$, be a scheme [4], for example, the scheme given from the derived categories $\mathrm{D}_{X}$, whose sheaves let $\mathrm{I}$, be coherent sheaves of ideals on $X$, then the transformation that we define is the morphism $\pi: \widetilde{X} \rightarrow X$, such that $\pi^{-1} \mid \mathrm{O}_{\widetilde{X}}$, is an invertible sheaf. Here $O_{\bar{X}}$, is the structure of the sheaf of $\widetilde{X}$.

Likewise, morphisms from schemes to affine schemes are completely understood in terms of ring homomorphisms by the following contravariant adjoint pair: For every scheme $X$, and every commutative ring $A$, we have a natural equivalence

$$
\operatorname{Hom}_{\text {Schemes }}(X, \operatorname{Spec}(A)) \cong \operatorname{Hom}_{\text {CRing }}\left(A, O_{X}(X)\right)
$$


since $A$, is an initial object in the category of rings, the category of schemes has as a final object. $\operatorname{Spec}(A)$, means the spectrum of a category of the commutative rings. The character Spec, is the functor "spectrum".

To our purposes the Axion particle must be a final element or object in the space $\operatorname{Spec}(A)$.

Def. 1. 1. The axion is an image of a spin manifold in the image of $\operatorname{Spec}(A)$, of the space-time.

This is not a spin manifold, however is the resulted of a transformation "blow up" 1 in the space-time of a spin manifold in QFT-frame. We define as image of a spin manifold under transformation rules in the space-time modeled as a complex Riemannian manifold of dimension $2 n$.

\section{An "Axion" of the Topological Characterizing of Energy Coverings in M.}

In superstring theory $[5,6]$, the construction of realistic phenomenological models requires dimensional reduction because the strings naturally propagate in a 10 -dimensional space whilst the observed dimension of space-time of the universe is 4 . Formal constraints on the theories nevertheless place restrictions on the compactified space in which the extra "hidden" variables live: when looking for realistic 4dimensional models with supersymmetry, the auxiliary compactified space must be a 6-dimensional Calabi-Yau manifold [7].

From a different point of view, and considering the time and space as fundamental entities that born from the universal covering of the Universe, that is the energy, we want establish the coupling of the space and time as only one, that creates a theoretical model particle to explain the movement phenomena and the creation of matter, this last, helping it with the quantum gravitation (for example: baryogenesis, TFT, etc) through other theories as mother gravity $[8,9,10,11]$, and other.

\section{Topological Quantum Diffeomorphisms in Field Theory}

Using the model in the figure 2 in [11], we consider the following: Let the corresponding non-compact Lagrangian submanifolds $L$, as homotopy of $L_{0}$, where $L_{0}$, is the Lagrangian submanifold before of the action field given by one particle, to know [12]:

$$
H W^{*}\left(L_{0}, L_{1}\right)=H\left(C W^{*}\left(L_{0}, L_{1}\right)\right)
$$

Using the conjecture of Fukaya considering $L=T_{x}^{*}$, a cotangent fiber, the $A_{\infty}$ - structure on $C W^{*}\left(L_{0}, L_{1}\right)$, should be quasi-isomorphic to the algebra $d g$ - structure on $C_{-*}\left(\Omega_{x}\right)$,

1 In mathematics, more specifically in algebraic geometry and the theory of complex manifolds, the coherent sheaves are a specific class of sheaves having particularly manageable properties closely linked to the geometrical properties of the underlying space. The definition of coherent sheaves is made with reference to a sheaf of rings that codifies this geometrical information. where $\Omega_{x}$, is the based loop space 2 of $(Z, x)$, where $Z$, is differentiable manifold which involves a normalized geodesic flow accord to their Poincaré section. This geodesic flow from a point of view strictly of the TFT, is conformed for the minimal trajectories with maximum benefit defined from a point of view of the variation principle. But this flow is created from the micro-states that can be measurable by the gauge fields [13] in the Hamiltonian space.

From the conjecture 1, given in [13] the movement is spread ramification from the energy-vacuum mechanism through paths of particle actions given by [14]

$$
\mathfrak{I}_{O_{c}}(\gamma)=\int_{M} O_{c}(\gamma(x)) d \gamma(x)
$$

The Bulnes's operator $O_{\mathrm{c}}$ [14], involves a connection $\sigma_{t}\left(X_{p}\right), \forall p \in M, X_{p} \in T_{p}(M) 3$ of the tangent bundle of the space of trajectories $\Omega(\Gamma) \subset \mathrm{R}^{3} \times I_{t}$, such that the map

$$
X \rightarrow \gamma_{X}(1)
$$

is a diffeomorphism from $U_{0} \subset T_{p}(M)$ to $U_{p}, \forall p \in M$, being $U_{0}, U_{p}$, open neighborhoods.

But, what is there with the quantum image of this diffeomorphism? Will be this also an image of other diffeomorphisms under the same topology or needs an extended or induced topology?

Then the corresponding paths in the quantum image $\mathrm{M}$, from the field actions given in (3) take the form to that energy states collection as:

$$
\mathfrak{I}_{O_{c}}(\varphi)=\int_{X(M)} O_{c}(\varphi(x)) d \varphi(x)
$$

preserving the same symplectic structure.

To it we need establish a correspondence between the wrapped Floer cohomology and the ordinary Floer cohomology given by $H^{*}(L)$, where the action functional (3) haves the same value that (5). The wrapped Floer cohomology that comes from of the energy that wraps the flow of geodesics $x:[0,1] \rightarrow M$, of the Hamiltonian $H$, and that generates the associated Floer cochain complex going from $L_{0}$, to $L_{1}$, having that 4

$$
C F^{*}\left(L_{0}, L_{1} ; \mathrm{H}\right) \cong C F^{*}\left(\varphi_{X}^{1}\left(L_{0}\right), L_{1}\right)
$$

2 In mathematics, the space of loops or (free) loop space of a topological space $X$, is the space of loops from the unit circle $S^{1}$, to $X$, together with the compact-open topology. $\Omega X=C\left(S^{1}, X\right)$,

That is, a particular function space. In particular $\Omega_{x}$, is the base loop space of $\Omega X$. In homotopy theory loop space commonly refers to the same construction applied to pointed spaces, i.e. continuous maps respecting base points. In this setting there is a natural "concatenation operation" by which two elements of the loop space can be combined. With this operation, the loop space can be regarded as a magma or even as an $A_{\infty}$-space. Concatenation of loops is not strictly associative, but it is associative up to higher homotopies.

3 Indeed, is the affine connection describes as:

$$
\sigma_{t}\left(X_{p}\right)=\nabla_{\gamma^{\prime}(t)} X=\exp _{p}(t X) .
$$

4 Here $\phi_{X}^{1}$ is the flow of geodesics $\gamma$, such that $\forall X$, a Hamiltonian vector field of $H$, is satisfied (4). 
which must suffer a little variation to create to the infinitum the wrapping such that given two $d g$-modules $E_{L}$, and $E_{Q}$, 5elements of a $d g$-category defined by $\mathrm{M}=\bmod (\mathrm{C})$. .where $\mathrm{C}$, is the Cěch complex defined by (where $d=|I|-1$ ).

$$
C=\bigoplus_{I} \Gamma\left(U_{I}\right)[-d]
$$

Theorem 3. 1. (Abbondandolo-Schwartz). Let $M=T^{*} Z$, be the cotangent bundle 6 of a closed oriented manifold, and take two cotangent fibers $L_{0}=T_{x_{0}}^{*} Z$ (that is to say, the obtained from $O_{\mathrm{c}}$ ) and $L_{1}=T_{x_{1}}^{*} Z$. Then

$$
H W^{*}\left(L_{0}, L_{1}\right) \cong H_{-*}\left(L_{x_{0}, x_{l}}\right)
$$

is the (negatively graded) homology of the space of paths in $Z$, going from $x_{0}$, to $x_{1}$.

Proof. [15].

Conjecture $4[16,17]$. Let $M=T^{*} Z$, be a cotangent fibre. Then the $d g$-module $\mathrm{E}_{L}$ (of Lagrangian submanifold $L$ ) is isomorphic to $\mathrm{E}_{L}$ in $H(\mathrm{M})$. Moreover, if, is simply connected G,7 gives rise to a quasi-isomorphism

$$
C_{-*}\left(\Omega_{x}\right) \cong C W^{*}(L, L) \rightarrow \operatorname{hom}_{m}\left(E_{L}, E_{L}\right)
$$

Proposition 3. 1 (F. Bulnes, M. Ramírez, L. Ramírez, O, Ramírez). The wrapping energy (spectrum) is characterized by the fields related by the diffeomorphism $C_{-*}\left(\Omega_{x}\right) \rightarrow \mathrm{W}(H)$, whose space of paths going from $\gamma(x)$, to $\phi(x)$, foreseen in (8). Then the ramification of field in this case is the connection to the operator $O_{c}: T M \rightarrow T^{*} M$.

Proof. [11].

\section{Wrapp Enery and the Space-Time}

Let $E$, be energy, $S$, space and $T$, time, and the corresponding sets $U_{S}^{E}$, and $U_{T}^{E}$, such that is satisfied the commutative elemental diagram:

$$
\begin{aligned}
& T \stackrel{\Lambda}{\longrightarrow} S \\
& \phi \downarrow \quad \downarrow \psi \\
& E \stackrel{I d}{\longrightarrow} E
\end{aligned}
$$

where in the space-time context, the application $\Lambda$, is a homomorphism of the Universe:

\footnotetext{
5 It's a $d g$ - module over a $d g$-algebra of Cěch cochains. This in other re-interpretation could be a sheaf of complexes.

6 The cotangent bundle $T^{*} Z$, of any $n$-dimensional manifold $Z$, is itself a manifold (of dimension $2 n$ ) and supports naturally an exact symplectic structure $\omega=d \lambda$. (This 1 -form $\lambda$, is sometimes called the Liouville form). There are several ways to construct an associated contact manifold, one of dimension $2 n-1$, one of dimension $2 n+1$.

$7 \mathrm{G}$ is the $A_{\infty}$-functor: $\mathrm{G}: \mathrm{W}(M) \rightarrow \mathrm{M}=\bmod (\mathrm{C})$,

Resulted of associates to any exact Lagrangian submanifold , which is Legendrian at infinity, a $d g-$ module $\mathrm{E}_{L}$, over $\mathrm{C}$.

Here a $d g-$ algebra over our coefficient field $\mathrm{K}$, with an augmentation $\varepsilon: \mathrm{C} \rightarrow \mathrm{K}$, whose kernel is denoted by $\mathrm{I}$. Then is possible equip the free tensor co-algebra $T(\mathrm{I}[1])$, with a differential and then dualizes it to a $d g-$ algebra $\mathrm{B}=T(\mathrm{I}[1])^{\vee}$.
}

$$
U_{T}^{S}=U_{T}^{E} U_{S}^{E}
$$

called the product of the time and space. Of fact is a homomorphism belonging to the homomorphism space given in the second member of (1) if we consider the spaces $E, S$, and $T$, as belonging to a spin manifold. From a point of view of the string theory, these spaces can be identified as orbifolds 8 of certain dimension each one.

Then $U_{T}^{S}$, is our Universe modeled as topological space. Of fact, this shapes an orbifold atlas. The opens $S$, and $T$, are included in $U_{T}^{S}$. We define our "axion" corresponding to the wrap energy under this frame:

Def. 3. 1. The corresponding axion $\eta$, to the wrap energy will be the universal coverings composition $T \rightarrow E$, and $S \rightarrow E$, in space-timeM.

From a point of view of the QFT, we can give the following simple definition:

Def. 3. 2. The corresponding axion to the wrap energy $\eta$, is a bi-particle that involve of an energy quanta of space (in the more elemental dimension an energy of length9) and an energy quanta of time.

We take $\eta=\left(\eta_{1}, \eta_{2}\right)$, in the energy open $E$. This is equivalent to consider the set of equivalence classes of closed minimal paths (loops) with base point the axion $\eta$. The space $\pi_{1}(E, \eta)$, is the fundamental group of the open energy $E, 10$ with base point the axion $\eta$.

Let the axions $\eta_{1}, \eta_{2} \in E$, and consider an affine connection whose trajectory let be minimal between them, that is to say, a ramification of minor consume of energy and time, then those do that the groups $\pi_{1}\left(E, \eta_{1}\right)$, and $\pi_{2}\left(E, \eta_{2}\right)$, let be isomorphic.

We suppose that the applications $T \rightarrow E$, and $S \rightarrow E$, are orbifolds universal coverings. Then $E$, is a wrapped energy resulted from these coverings [11].

Now supposing $S$, and $T$, are connected by minimal trajectories, and let $\mu: E \rightarrow T$, and $v: E \rightarrow S$, the two homotopic [19] equivalences given in the diagram (10) then

$$
\varphi_{*}: \pi(T, \mu(\eta)) \rightarrow \pi(S, v(\eta))
$$

is an isomorphism.

Indeed, considering the following diagrams this property can be proved:

8 In the topology, geometry, and geometric group theory an orbifold (for "orbit-manifold") is a generalization of a manifold. It is a topological space (called the underlying space) with an orbifold structure.

An n-dimensional orbifold is a Hausdorff topological space $X$, called the underlying space, with a covering by a collection of open sets $U_{i}$, closed under finite intersection. For each $U_{i}$, there is

an open subset $V_{i}$, of $\mathrm{R}^{n}$, invariant under a faithfull linear action of a finite group $\Gamma_{i}$

a continuous map $\varphi_{i}$, of $V_{i}$, onto $U_{i}$, invariant under $\Gamma_{i}$, called an orbifold chart, which defines a homeomorphism between $V_{i} / \Gamma_{i}$, and $U_{i}$.

9 Following some ideas given in [18] we consider the length between points of space-time in the frame as discrete. Of fact is calculated in [18].

10 There are several ways to define the orbifold fundamental group. More sophisticated approaches use orbifold covering spaces or classifying spaces of groupoids. 


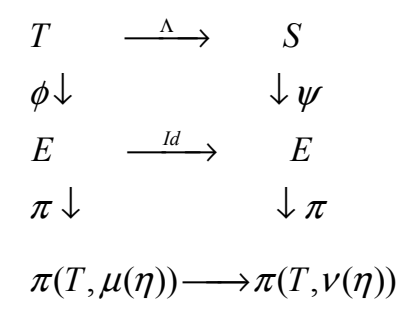

The open sets $E, S$, and $T$, are connected for minimal trajectories, thus is possible to say that $T$, is a covering of $E$, also $S$, is a covering of $E$, being taken the continuous applications and on $\varphi: T \rightarrow E$, and $\psi: S \rightarrow E$, such that to each axion $\eta \in E$, there exist an open neighborhood $U_{\eta}$, that satisfy: $\varphi^{-1}\left(U_{\eta}\right)=\bigcup_{j} u_{j}$, with $u_{j}$, open sets $\forall j$, and the corresponding to $\psi$.

$\varphi_{u_{j}}: u_{j} \rightarrow U_{\eta}$, is a homomorphism $\forall j$. Also $\psi_{u_{k}}: u_{k} \rightarrow V_{\eta}$, such that $u_{j} \cap u_{k} \neq \varnothing$.

Here $U_{\eta}$, and $V_{\eta}$, are regular neighborhoods and the open sets $u_{j}$, and $u_{k}$, are folds ${ }^{11}$.

Newly consider the axion $\eta$. Given a subgroup, of, there is a connected covering by minimal trajectories called universal covering, as was signed in the sections II, III, and IV, of this work. Now remember that is called to the open sets $E, S$, and $T$, the energy, time and space, and, the Universe as topological space $E$, whose spectrum is the space $U_{s}^{T}$.

Then using the Seifert-Van Kampen theorem [20] we consider the following characteristics:

1). $E, \quad S$, and $T$, opens and connected by minimal trajectories of the topological spaces $U_{S}^{E}$, and $U_{S}^{E}$, such that

$$
E \cup T=U_{T}^{E}, \quad E \cap T \neq \varnothing,
$$

and

$$
E \cup S=U_{S}^{E}, \quad E \cap S \neq \varnothing,
$$

where $E \cap T$, and $E \cap T$, also connected by minimal trajectories. Let $\eta$, be the bi-particle called axion of wrap energy such that $\eta \in E \cap T$, and $\eta \in E \cap S$, as base to the fundamental groups.

2. Let $K_{S}^{E}$, and $K_{T}^{E}$, the arbitrary groups and consider the following homomorphisms $\rho_{1}, \rho_{2}, \rho_{3}, \theta_{1}, \theta_{2}$, and $\theta_{3}$, and such that the following diagram commute:

11 In string theory the orbifolds are generalizations of the notion of manifold that allow the presence of the points whose neighborhoods are diffeomorphic to a quotient of $\mathrm{R}^{n}$, by a finite group, i.e. $\mathrm{R}^{n} / \Gamma$. A QFT defined on an orbifold becomes singular near the fixed points of G. However string theory requires us to add new parts of the closed string Hilbert space, namely the twisted sectors where the fields defined on the closed strings are periodic up to an action from G. Orbifolding is therefore a general procedure of string theory to derive a new string theory from an old string theory in which the elements of $G$ have been identified with the identity. This is our case that we are using.

$D$ - branes, propagating on the orbifolds are described, at low energies, by gauge theories defined by the quiver diagrams. Open strings attached to these $D-$ branes have no twisted sector, and so the number of open string states is reduced by the orbifolding procedure.

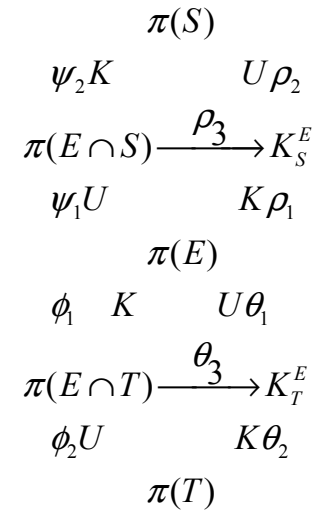

By (11) there are homomorphisms:

$$
\sigma: \pi\left(U_{T}^{E}\right) \rightarrow K_{T}^{E}, \quad \tau: \pi\left(U_{S}^{E}\right) \rightarrow K_{S}^{E}
$$

such that the following diagram commute:

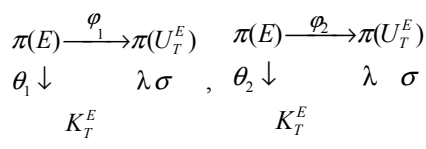

and

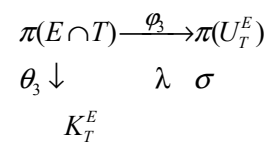

Also the corresponding to $S$,

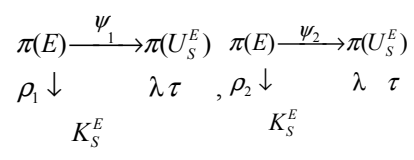

and

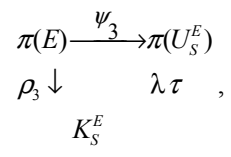

Then

$$
K_{T}^{E} K_{S}^{E}=K_{S}^{T}
$$

where $\varphi_{i}, \psi_{i}$, are induced homomorphism for inclusions. All before development is applied in the case when $T$, and $S$, are considered as bases to the executed process and observe the homomorphisms between $E$, and $T$, and also $E$, and $S$. Then $E$, and $T$, as also $E, S$, are mutually covered, and the elements for that are constituted, the axions $\eta$, are their fundamental elements of the open sets of the Universe $U_{T}^{S}$.

\section{Results}

From the proposition of section 4, the important fact of that there exists a wrapping energy that gives support to the topological diffeomorphisms in QFT, to the space and the 
time, give step to that their ramification of field (the connection of the loop space $\left.C_{-*}\left(\Omega_{x}\right)\right)$ is a connection obtained under the following scheme:

$$
\begin{array}{ccc}
O_{c}(\varphi) \in H\left(\bmod f\left(C_{-*}(\Omega Z)\right)\right) \stackrel{R^{-1}}{\longrightarrow} & H(M) \longrightarrow & C \\
\downarrow & \downarrow \text { embb } & \downarrow \\
C_{-*}\left(\Omega_{x}\right) & \stackrel{\text { Diff }}{\longrightarrow} & W(H) \ni \varphi \stackrel{G}{\longrightarrow} M
\end{array}
$$

where the distinguished diffeomorphism $C_{-*}\left(\Omega_{x}\right) \rightarrow \mathrm{W}(H)$, is demonstrated in the proposition. The descendent mappings are considered the ramifications of field that under transformations of energy can be topological spaces created by the corresponding flows of geodesics, strings or $D-$ branes.

Then using the scheme on functoriality of rings planted in (1) considering the connection scheme to the ramified field in the space-time $M$, we can enounce the following generalizing of (11) given by the following Theorem:

Theorem (F. Bulnes) 5. 1. If we consider the category $M_{\mathrm{K}_{F}}(\mathrm{~g}, \mathrm{Y})$, then a scheme of their spectrum $\mathrm{W}(H)$, where $\mathrm{Y}$, is a Calabi-Yau manifold comes given as:

$$
\operatorname{Hom}_{\hat{\mathrm{g}}}\left(X, \mathrm{~V}_{\text {critical }}^{\text {Def }}\right) \cong \operatorname{Hom}_{\mathrm{Loc}_{L_{G}}}\left(\mathrm{~V}_{\text {critical }}, M_{\mathrm{K}_{F}}(\hat{\mathrm{g}}, \mathrm{Y})\right)
$$

Proof. [2, 3].

Applying the before theorem to the context of our coverings and axions we have the following corollary:

Corollary (M. Ramírez, L.Ramírez, O. Ramírez, F. Bulnes) 5. 1. We have the following scheme:

$$
\operatorname{Hom}_{\hat{\mathrm{so}(1,3)}}\left(U, K_{T}^{S}\right) \cong \operatorname{Hom}_{\text {CRing }}\left(E, \phi_{*}(\pi(T, \mu(\eta)))\right)
$$

where $\phi \in \mathrm{W}(H)$, and their pull-back is $\phi_{*}$.

\section{Conclusions}

There is a committed hypothesis that emerges from this analysis, we attach, the analyzed the interim lapse between the beginning and end; is what we know and is acquaintance; in this context, the axion concerns and refer the simultaneous action of space-time through of the development of elements of energy, certain orbifolds that born of a spin manifold. The axion $\eta$, is the minimal particle created to start of a covering transformation of two fundamental aspects of the apparent Universe and helps to define with major precision the movement and the matter, where this last stay defined with help of the gravity theories of ramified field.

The fundamental groups $\pi(T, \mu(\eta))$, and $\pi(S, \nu(\eta))$, used, likewise the coverings and uprisings (homotopies), constitute the operative elements of this theory. The central idea of this research consists in consider, without being risky, that the Universe (at least in the quantum level) can be modeled by axions $\eta$, where all other, only are manifestations of these, and can be added to other major aspects as the thermodynamic type, the existence of singularities of the space-time and their effects to define a complex spin manifold that explain the born of all ten dimensions that require other theories as the string theory, since the entity particle and field are dual concepts in the second postulating of the QFT.

Their spectrum can be viewed an aspect of one version of the Floer cohomology and their Lagrangians that are the object in the other class of the homomorphism that include the ring structure.

\section{Acknowledgements}

We are grateful with the Lead Guest Editor in this special issue for their help and direction in this research.

\section{References}

[1] F. Bulnes, "Design of Quantum Gravity Sensor by Curvature Energy and their Encoding," Proc. IEEE-UK, London, UK, 2014.

[2] F. Bulnes, "Geometrical Langlands Ramifications and Differential Operators Classification by Coherent $D$-Modules in Field Theory," Journal of Mathematics and System Science, 3, no. 10, 2013, USA, pp491-507.

[3] Bulnes, F. (2014) Derived Categories in Langlands Geometrical Ramifications: Approaching by Penrose Transforms. Advances in Pure Mathematics, 4, 253-260. doi: 10.4236/apm.2014.46034.

[4] D. Eisenbud: J. Harris (1998). The Geometry of Schemes. Springer-Verlag, USA.

[5] Blumenhagen, Ralph; Lüst, Dieter; Theisen, Stefan (2012), Basic Concepts of String Theory, Theoretical and Mathematical Physics, Springer, p. 487, "Orbifolds can be viewed as singular limits of smooth Calabi-Yau manifolds".

[6] M. Green, J. Schwartz and E. Witten, Superstring theory, Vol. 1 and 2, Cambridge University Press, 1987.

[7] N. Hitchin (2003), "Generalized Calabi-Yau manifolds", The Quarterly Journal of Mathematics 54 (3): 281-308.

[8] M. A. Ramírez, L. Ramírez, A. Camarena, The Mother Gravity, Procc. Appliedmath 2, November, México, City, 2006.

[9] M. A. Ramírez, L. Ramírez, A. Camarena, The Mother Gravity II: Genesis Dialectic, Procc. Appliedmath 3, October, México, City, 2007.

[10] M. A. Ramírez, L. Ramírez, A. Camarena, The Mother Gravity III: walking for Rams, Procc. Appliedmath 3, November, México, City, 2008.

[11] M. Ramírez, L. Ramírez, O. Ramírez, F. Bulnes, "Field Ramifications: The Energy-Vacuum Interaction that Produces Movement," Journal on Photonics and Spintronics, Vol. 2, no. 3, USA, 2013, pp4-11.

[12] J. Milnor, "On spaces having the homotopy type of a CW-complex" Trans. Amer. Math. Soc. 90 (1959), 272-280.

[13] M. Ramírez, L. Ramírez, O. Ramírez, F. Bulnes, "Field Ramifications: The Energy-Vacuum Interaction that Produces Movement," Journal on Photonics and Spintronics, Vol. 2, no. 3, USA, 2013, pp4-11. 
[14] F. Bulnes (2013). Quantum Intentionality and Determination of Realities in the Space-Time Through Path Integrals and Their Integral Transforms, Advances in Quantum Mechanics, Prof Paul Bracken (Ed.), ISBN: 978-953-51-1089-7, InTech, DOI: 10.5772/53439. Available from: http://www.intechopen.com/books/advances-in-quantum-mec hanics/quantum-intentionality-and-determination-of-realities-i $\mathrm{n}$-the-space-time-through-path-integrals-and-t

[15] A. Abbondandolo, M. Schwarz, "Floer homology of cotangent bundle and the loop product," Geom. Top. 14 (2010), no. 3, $1569-1722$.

[16] K. Fukaya, Floer Homology and Mirror Symmetry I, Department of Mathematics, Faculty of Science, Kyoto University, Kitashirakawa, Kyoto, 606-8224, Japan.
[17] A. Kapustin, M. Kreuser and K. G. Schlesinger, Homological mirror symmetry: New Developments and Perspectives, Springer. Berlin, Heidelberg, 2009.

[18] F. Bulnes, (2013) Mathematical Nanotechnology: Quantum Field Intentionality. Journal of Applied Mathematics and Physics, 1, 25-44. doi: 10.4236/jamp.2013.15005.

[19] R. M. Switzer, Homotopy and Homology. Springer, 2nd Edition, 1975.

[20] J. G. Hocking, G. S. Young., Topología. Editorial Reverte S.A. Barcelona, España. 1966. 